\title{
A novel whole yeast-based subunit oral vaccine against Eimeria tenella in chickens
}

1 Francesca Soutter ${ }^{1,2}$. Dirk Werling ${ }^{* 1}$ Matthew Nolan $^{1,3}$, Tatiana Küster $^{1,4}$, Elizabeth Attree ${ }^{1}$,

2 Virginia Marugán-Hernández ${ }^{1}$, Sungwon Kim ${ }^{1,5}$ Fiona M. Tomley $^{1}$, Damer P. Blake*1

$3{ }^{1}$ Department of Pathobiology and Population Sciences, Royal Veterinary College, North Mymms,

4 Hertfordshire, AL9 7TA, UK

$5 \quad{ }^{2}$ Current address: Scotland's Rural College, Edinburgh, EH9 3JG

$6 \quad{ }^{3}$ Current address: University of Cambridge, Cambridge, CB3 0JX, UK

74 . Current address: Boehringer Ingelheim Animal Health, Binger Str. 173, 55216, Ingelheim am

8 Rhein, Germany

$9 \quad 5$. Current address: Touchlight Genetics Ltd, Hampton, UK

10

$11 *$ Correspondence:

12 Damer Blake and Dirk Werling

13 dblake@rvc.ac.uk/dwerling@rvc.ac.uk

14 Keywords: Eimeria tenella ${ }_{1}$; Saccharomyces cerevisiae $_{2}$; vaccine $_{3}$; chicken $_{4}$; yeast 5

Abstract

Cheap, easy-to-produce oral vaccines are needed for control of coccidiosis in chickens to reduce the impact of this disease on welfare and economic performance. Saccharomyces cerevisiae yeast expressing three Eimeria tenella antigens were developed and delivered as heat-killed, freeze-dried whole yeast oral vaccines to chickens in four separate studies. After vaccination, E. tenella replication was reduced following low dose challenge (250 oocysts) in Hy-Line Brown layer chickens $(\mathrm{p}<0.01)$. Similarly, caecal lesion score was reduced in Hy-Line Brown layer chickens vaccinated using a mixture of S. cerevisiae expressing EtAMA1, EtIMP1 and EtMIC3 following pathogenic-level challenge (4,000 E. tenella oocysts; p<0.01). Mean body weight gain post-challenge with 15,000 E. tenella oocysts was significantly increased in vaccinated Cobb500 broiler chickens compared to mock-vaccinated controls $(\mathrm{p}<0.01)$. Thus, inactivated recombinant yeast vaccines offer cost-effective and scalable opportunities for control of coccidiosis, with relevance to broiler production and chickens reared in low-and middle-income countries (LMICs).

\section{Introduction}

Coccidiosis, a disease of the gastrointestinal tract caused by Eimeria parasites, is a considerable burden to the poultry industry economically, estimated to cost over $£ 10$ billion per year (1), and in terms of chicken health and welfare, causing diarrhoea and ill-thrift. Existing vaccines that consist of controlled oral doses of live or live-attenuated parasites are efficacious and widely used in egg-laying and breeder chicken populations. However, despite achieving levels of protection comparable to anticoccidial drugs $(2,3)$, uptake in the broiler chicken sector has been limited, at least in part 
because the cost of vaccines is relatively high (1). Furthermore, the current live anticoccidial vaccines can only be produced by controlled infection of specific-pathogen-free chickens, creating an inherent limit on productive capacity and questions around the ethical use of chickens for production of vaccines. Even if demand for existing vaccines increases, it is unlikely that the production of liveattenuated vaccines could be scaled up sufficiently to supply the entire broiler sector. Thus, novel oral vaccines against coccidiosis in chickens would provide a much-needed alternative to these current vaccines as well as in-feed anticoccidial drugs.

Recombinant protein vaccines, both subunit and "live" recombinant, targeting Eimeria species have long been examined as an alternative to current vaccines, and a number of immunoprotective antigens, such as Apical Membrane Antigen 1 (AMA1), Immune Mapped Protein 1 (IMP1) and Microneme Protein 3 (MIC3) have been identified (reviewed in Blake et al., 2017). Reduced parasite replication and gut pathology have been demonstrated when these antigens were given as subunit vaccines such as E.coli expressed recombinant proteins, DNA vaccines or administered using vectored approaches such as expression in transgenic Eimeria. For example, proof-of-concept vaccination trials administering three sub-cutaneous or intramuscular doses of recombinant protein have been successful at reducing parasite replication (faecal oocyst count) following low dose parasite oral challenge (250 oocysts) (4-6). However, the delivery of recombinant vaccines by intramuscular injection or other individual bird-by-bird approaches is unsuitable for large scale vaccination of chickens in the field, inhibiting commercialisation. Oral recombinant subunit vaccines that stably express candidate immunoprotective antigens provide an easier method of delivery to chickens in all environments and are therefore highly desirable.

Yeasts, such as Saccharomyces cerevisiae, have long been utilized for the production of recombinant soluble proteins for use in applications such as vaccination(7). More recently, S. cerevisiae has been used for oral vaccine delivery as whole recombinant yeast (live or killed), combining multiple purposes such as antigen expression and carriage, as well as being its own adjuvant. In addition to convenience for vaccine delivery, oral administration of recombinant $S$. cerevisiae supports direct vaccine interaction with the mucosal surface of the gastrointestinal tract, where Eimeria parasites invade, and can stimulate an immune response through pattern recognition receptors (PRRs), negating the need for adjuvants (8). Furthermore, S. cerevisiae and its cell wall derivatives are already used as feed supplements in the poultry sector and some studies have demonstrated its efficacy in reducing reproduction of Eimeria parasites (9) as well as improved production parameters during infection $(10,11)$, even without the addition of a vaccinal antigen. Previous studies of $S$. cerevisiae expressing microneme proteins have demonstrated efficacy in reducing oocyst output and caecal lesions following vaccination with live oral yeast $(12,13)$. Finally, recombinant yeast lines can be inactivated, permitting Generally Regarded as Safe (GRAS) status that may improve industry and social acceptance (14).Furthermore it has been suggested that inactivation could enhance antigenicity of yeast lines by altering distribution and exposure of structures such as $\beta-1,3$ glucan on the yeast cell surface (15).

The aim of this study was to produce a whole yeast vaccine using recombinant S. cerevisiae expressing the Eimeria tenella antigens EtAMA1, EtIMP1 and EtMIC3, and to assess efficacy in reducing challenge-induced parasite replication and pathognomonic lesions in the caeca in vivo following yeast inactivation and oral vaccination. The efficacy of vaccination using yeast expressing single E. tenella antigens or combinations of yeast expressing each antigen are assessed and compared to E.coli expressed, purified recombinant protein and mock live oocyst vaccination following low or high dose parasite challenge. 


\section{Materials and Methods}

\subsection{Ethics statement}

This study was performed under a UK Home Office License according to the Animals in Scientific Procedures Act 1986 (ASPA). Procedures were approved by the Royal Veterinary College (RVC) Animal Welfare Ethical Review Body (AWERB).

\subsection{Vaccine antigens}

Three E. tenella (Houghton strain) antigens were selected for surface expression on $S$. cerevisiae; the ectodomain of Apical Membrane Antigen 1 (EtAMA1), Immune Mapped Protein 1 (EtIMP1) and repeat 3 from Microneme Protein 3 (EtMIC3), one of three identical Microneme Adhesive Region (MAR) domains contained within the ectodomain (16). DNA sequences for EtAMA1, EtIMP1 and EtMIC3 were obtained from Genbank, (accession numbers LN609976.1, FN813229.2 and FJ374765.1 respectively). Codon optimisation for S. cerevisiae was performed using the codon usage database (http://www.kazusa.or.jp/codon) for EtAMA1 and EtIMP1, but synthesis was unsuccessful for EtMIC3.

\subsection{Cloning strategies applied}

\section{$97 \quad$ Study 1}

The E. tenella AMA1 ectodomain coding sequence, representing amino acids 24-446, was combined with a 3' codon optimised citrine tag (EtAMA1Cit) in the first study for assessment of protein expression. In parallel, the E. tenella IMP1 coding sequence (amino acids 2-387) was also combined with a 3' citrine tag (EtIMP1Cit). DNA constructs were synthesised by Eurofins Genomics (Luxembourg) within a pEX-K4 (EtAMA1Cit) or pMK-RQ (EtIMP1Cit) plasmid with appropriate restriction enzyme sites for cloning into pYD1 yeast display plasmid vector (Invitrogen, Thermofisher Scientific, Waltham, MA, USA). A NotI restriction site was included between each antigen sequence and the 3' citrine tag. Attempts to synthesise the EtMIC3 R3 sequence were unsuccessful and thus this antigen was not included in Study 1.

107 Synthesised antigen constructs were transformed into competent XL-1 Blue Escherichia coli cells 108 (Agilent Technologies, Santa Clara, CA, USA) by electroporation, according to manufacturer's instructions and pipetted on to selective Lysogeny Broth (LB) agar plates (50 $\mu \mathrm{g} \mathrm{mL}^{-1}$ kanamycin, 20 $\mathrm{mM}$ IPTG, $80 \mu \mathrm{g} \mathrm{mL}^{-1} \mathrm{X}$-gal, all Sigma Aldrich, St Louis, MO, USA) and incubated overnight at 37 ${ }^{\circ} \mathrm{C}$. Blue/white colony screening was performed, and selected colonies grown in LB broth $(50 \mu \mathrm{g}$ $\mathrm{mL}^{-1}$ kanamycin) overnight at $37^{\circ} \mathrm{C}$ with shaking at $160 \mathrm{rpm}$. Plasmid DNA was prepared from overnight cultures using the QIAprep Spin Miniprep kit (QIAGEN, Hilden, Germany) according to

114 manufacturer's instructions.

115 Restriction digest of pEX-K4 plasmid DNA containing EtAMA1Cit or was performed using Bam HI and Xho I (New England Biolabs, Ipswich, MA, USA) to extract tagged antigen coding sequences for cloning into pYD1 plasmid vector. Restriction digest of pYD1 plasmid was performed with the same restriction enzymes. Restriction digest of pMK-RQ plasmid DNA containing EtIMP1 was performed using Bam HI and Not I restriction enzymes (New England Biolabs). Restriction digest of pYD1- 
but retain the citrine tag. Restriction digest products were visualised by gel electrophoresis on a 0.8

$\%$ agarose gel and bands cut out using a scalpel blade. Gel extraction was performed using the QIAquick gel extraction kit (QIAGEN) according to manufacturer's instructions and concentration quantified by spectrophotometry using the DS-11 FX spectrophotometer (Denovix, Wilmington, DE, USA). Ligation was performed on gel purified insert and digested plasmid with T4 Ligase (Promega, Madison, WI, USA) according to manufacturer's instructions, ligation reactions were transformed into XL-1 Blue E. coli cells (Agilent Technologies) by electroporation as before and pipetted on to selective LB agar plates (100 $\mathrm{g} \mathrm{mL}^{-1}$ Ampicillin, $20 \mathrm{mM} \mathrm{IPTG,} 80 \mu \mathrm{g} \mathrm{mL}^{-1} \mathrm{X}$-gal, all Sigma Aldrich). Blue/white colony screening was performed, and selected colonies sent for Sanger sequencing at Eurofins Genomics to confirm correct integration of EtAMA1Cit into the pYD1 plasmid or EtIMP1 into the pYD1-Cit plasmid. Following confirmation by sequencing, plasmid DNA was prepared from overnight cultures as before and then used for yeast transformation. Competent $S$. cerevisiae EBY100 strain cells (Thermofisher Scientific) were transformed using the S.c.

EasyComp $^{\mathrm{TM}}$ Transformation Kit (Thermofisher Scientific) according to manufacturer's instructions. Transformants were grown on minimal dextrose plates supplemented with $1 \%$ leucine and $2 \%$ glucose at $30{ }^{\circ} \mathrm{C}$ for 3-5 days. Empty (undigested) pYD1 plasmid DNA was also transformed into $S$. cerevisiae EBY100 strain.

\section{Studies 2,3 and 4}

139 To optimize antigen expression in yeast, plasmids were re-constructed without the citrine tag.

140 EtAMA1 and EtIMP1 coding sequences (codon-optimised for yeast) were excised from the constructs generated in study 1 by restriction digest with BamHI and NotI (New England Biolabs). Cloning of the untagged EtAMA1 and EtIMP1 coding sequences into pYD1 was then performed as described for study 1 .

144 The target E. tenella MIC3 R3 (EtMIC3) sequence (not codon-optimised for yeast) had previously 145 been cloned into pET22b plasmid (MerckMillipore, Burlington, MA, USA) and transformed into XL-1 Blue E. coli cells (Agilent Technologies) in another study and stored in glycerol at $-80{ }^{\circ} \mathrm{C}(16)$. A sub-sample was streaked on to selective LB agar plates $\left(100 \mu \mathrm{g} \mathrm{mL}^{-1}\right.$ Ampicillin) and incubated overnight at $37^{\circ} \mathrm{C}$. Selected colonies grown in LB broth $\left(100 \mu \mathrm{g} \mathrm{mL}{ }^{-1}\right.$ Ampicillin $)$ overnight at $37^{\circ} \mathrm{C}$ with shaking at $160 \mathrm{rpm}$. Plasmid DNA was prepared from overnight cultures using the QIAprep Spin Miniprep kit (QIAGEN) according to manufacturer's instructions.

PCR was used to amplify EtMIC3 DNA from the pET22b MIC3 plasmid using primers (F: GCTATCGGATCCCAAGCCGTTCCAGAGG, R:

CTGCGAGAATTCGCCACTTGGATCTTCCGTT, $0.4 \mu \mathrm{M}$ final concentration, Sigma Aldrich) that incorporated appropriate restriction enzyme sites (Bam HI and Eco RI) for cloning into pYD1. Each $50 \mu \mathrm{L}$ reaction contained $5 \mu \mathrm{L}$ High Fidelity PCR Buffer 10x, $1 \mu \mathrm{L}$ dNTP mix $(0.2 \mathrm{mM}$ final concentration each), $2 \mu \mathrm{L} \mathrm{MgSO} 4$ ( $2 \mathrm{mM}$ final concentration) and $0.2 \mu \mathrm{L}$ Invitrogen ${ }^{\mathrm{TM}}$ Platinum $^{\mathrm{TM}}$ Taq DNA Polymerase High Fidelity(1IU; all ThermoFisher Scientific). heated to $94{ }^{\circ} \mathrm{C}$ for $1 \mathrm{~min}$, followed by 35 cycles consisting of $94{ }^{\circ} \mathrm{C}$ for $15 \mathrm{~s}, 55^{\circ} \mathrm{C}$ for $30 \mathrm{~s}$ and 68 extracted from the gel as before. Restriction digest of EtMIC3 PCR product was performed using Bam HI and Eco RI restriction enzymes (New England Biolabs) to extract antigen coding sequence for cloning into pYD1 plasmid vector. Cloning of EtMIC3 into pYD1 was then performed as described for study 1. 
165 Transformation of pYD1 plasmids containing EtAMA1, EtIMP1 and EtMIC3 into S. cerevisiae

166 EBY100 yeast was carried out as described above for study 1.

\subsection{Production of yeast and confirmation of expression}

168 Saccharomyces cerevisiae EBY100 yeast transformed with pYD1 including E. tenella antigen coding 169 sequences (Study1: EtAMA1Cit, EtIMP1Cit; Studies 2-4: EtAMA1, EtIMP1, EtMIC3) were grown 170 on minimal dextrose plates (0.67\% Yeast Nitrogen Base (YNB), $1.5 \%$ agar, both Sigma Aldrich) supplemented with $1 \%$ leucine (Sigma Aldrich) and $2 \%$ glucose (Sigma Aldrich) at $30{ }^{\circ} \mathrm{C}$ for 3-5 days. Single colonies were inoculated into YNB-CAA $(0.67 \%$ YNB, $0.5 \%$ Casamino acids (CAA), Calbiochem, San Diego, CA, USA) medium containing $2 \%$ glucose and grown overnight at $30{ }^{\circ} \mathrm{C}$ plus shaking $200 \mathrm{rpm}$ in an orbital shaker (New Brunswick ${ }^{\mathrm{TM}}$ Excella ${ }^{\circledR}$ E24 Shaker). Overnight cultures were centrifuged at 4,000 $\times \mathrm{G}$ for $10 \mathrm{~min}$ at room temperature and resuspended in YNBCAA containing $2 \%$ galactose (Sigma Aldrich) to an $\mathrm{OD}_{600}$ of 1.0 to induce protein expression. Yeast were cultured for $24 \mathrm{~h}$ at $30{ }^{\circ} \mathrm{C}$ plus shaking $200 \mathrm{rpm}$ in an orbital shaker.

Confirmation of protein expression $24 \mathrm{~h}$ post-induction was assessed by antibody staining and flow cytometry. A volume of yeast equal to an OD600 of 2.0 was pelleted by centrifugation at $6000 \times \mathrm{G}$ for 3 min and washed in PBS. Cell pellets were then incubated with a mouse Anti-V5 tag monoclonal antibody (1.2 $\mathrm{mg} \mathrm{mL}^{-1}$; Thermofisher Scientific) in PBS $0.1 \%$ BSA for $45 \mathrm{~min}$ at $4{ }^{\circ} \mathrm{C}$. Cells were then washed twice in PBS then suspended in goat anti-mouse IgG (H\&L) cross-adsorbed secondary antibody Alexa Fluor 488 (2 $\mathrm{mg} \mathrm{mL}^{-1}$; Thermofisher Scientific) in PBS 0.1\% BSA for 45 min at 4 ${ }^{\circ} \mathrm{C}$. Cells were then washed twice with PBS and then resuspended in FACSFlow (Becton Dickinson, Franklin Lakes, NJ, USA) prior to analysis using a FACSCalibur (Becton Dickinson). Expression was analysed using the FlowJo software package (V10, FlowJo LLC, Ashland, OR, USA), by comparing expression of the V5 tag, expressed at the 3' end of the antigen coding sequence, at $24 \mathrm{~h}$ post-induction compared with the staining obtained prior to induction.

189 For study 1 , yeast (24 h post-induction) were counted using the TC20 ${ }^{\mathrm{TM}}$ automated cell counter (BioRad Hercules, CA, USA). Cells were centrifuged at 4,000 $\times \mathrm{G}$ for $10 \mathrm{~min}$ and resuspended in PBS (Thermofisher). Yeast cells were heat-treated at $56^{\circ} \mathrm{C}$ for $1 \mathrm{~h}$, pelleted and then freeze-dried overnight using a Lyodry compact (Mechatech Systems Ltd, Bristol, UK) and stored at $-20{ }^{\circ} \mathrm{C}$ until oral inoculation into chickens. $1.7 \times 10^{7}$ yeast cells were resuspended and delivered to each chicken by oral gavage in $100 \mu \mathrm{L}$ of PBS. For chickens receiving both EtAMA1 and EtIMP1, $50 \mu \mathrm{L}$ of each was combined and $1.7 \times 10^{7}$ yeast cells in total were delivered to each chicken in $100 \mu \mathrm{L}$ of PBS.

For studies 2, 3 and 4, yeast (24 h post-induction) were counted as described. Cells were centrifuged at 4,000 $\times \mathrm{G}$ for $10 \mathrm{~min}$ and resuspended in PBS (Thermofisher) to a concentration of $1.5 \times 10^{7}$ cells $\mathrm{mL}^{-1} .1 \mathrm{ml}$ aliquots were heat-treated at $95^{\circ} \mathrm{C}$ for $2 \mathrm{~min}$, pelleted and then freeze-dried overnight as before. Freeze-dried yeast were stored at $4{ }^{\circ} \mathrm{C}$ and then resuspended in individual doses of $600 \mu \mathrm{L}$ PBS $24 \mathrm{~h}$ prior to oral inoculation of yeast into chickens. For chickens receiving all three yeast expressing antigens, each was resuspended in $600 \mu \mathrm{L}$ and then $200 \mu \mathrm{L}$ of each yeast was mixed in one microcentrifuge tube for oral dosing. (at two concentrations: $1.5 \times 10^{7}$ cells $\mathrm{mL}^{-1}$ and $4.9 \times 10^{8}$ cells $\mathrm{mL}^{-1}$ ) on to minimal dextrose plates supplemented with $1 \%$ leucine and $2 \%$ glucose at $30{ }^{\circ} \mathrm{C}$. Killing was confirmed by the absence of growth after 5 days incubation. Second, $\sim 1.5 \times 10^{7}$ heat killed yeast cells were diluted in $5 \mathrm{ml}$ YNBCAA medium containing $2 \%$ glucose and grown for five days at $30{ }^{\circ} \mathrm{C}$ plus shaking $200 \mathrm{rpm}$ in an 
orbital shaker. Growth was assessed by spectrophotometry, comparing $\mathrm{OD}_{600}$ of heat killed and sterile (i.e. no yeast) broths.

\subsection{Animals}

211 For studies 1,2 and 3, female Hy-line Brown layer chickens were purchased at day of hatch from Hy-

212 line UK Ltd (Studley, UK). All layer chickens were vaccinated against Marek's disease (Nobilis

213 Rismavac+CA126, MSD, Milton Keynes, UK) at the hatchery prior to the start of the study. Layer

214 chickens were fed a commercial organic starter feed, free from anticoccidial drugs. For study 4,

215 Cobb500 broiler chickens were purchased from P. D. Hook (Hatcheries) Ltd. (Cote, UK), at day of hatch. Cobb500 broiler chickens were vaccinated against infectious bronchitis (Nobilis IB H120, MSD Animal Health, Milton Keynes, UK). Broiler chickens were fed ad-lib throughout the study receiving starter feed from day 0-8, grower feed from day 9-18 and finisher feed from day 19 until the end of the study, all feeds were free from anticoccidial drugs (Target feeds, Whitchurch, Shropshire, UK) (Supplementary Table 1).

\subsection{Parasites}

The E. tenella Houghton $(\mathrm{H})$ reference strain was used in this study (17). Parasites were passaged through chickens at the Royal Veterinary College as originally described by (18) and were used within three months of sporulation.

\subsection{Experimental design}

\section{Study 1 (Low dose challenge-layer chickens)}

227

228

229

230

231

232

233

234

235

236

237

238

239

240

241

242

243

A low parasite dose challenge study was used to assess vaccine efficacy against E. tenella replication, recognising that quantification of replication following higher doses can be complicated by the Eimeria crowding effect (19). Forty-eight female Hy-Line Brown layer day of hatch chicks were weighed and divided into eight groups of six to seven chicks, so that each group contained a mixture of chickens of approximately the same weight (Supplementary Table 2), each group was housed in a separate cage. At day 7 all birds were wing tagged for identification of individual chickens. Four groups received an oral yeast vaccine by oral gavage every 3-4 days from day 7 of age (five doses per chicken in total); empty vector (pYD1 only), pYD1-EtAMA1Cit, pYD1-EtIMP1Cit or an equal mixture of pYD1-EtAMA1Cit and pYD1-EtIMP1Cit. One group received a low dose live oocyst "vaccine" of 100 E. tenella oocysts by oral inoculation at day 7 of age(20), although vaccine recycling was much reduced by accommodation in wire floored cages preventing chicken access to most faecal material. One group received E.coli expressed recombinant EtIMP1 protein, prepared as previously described (6), by intramuscular injection at day 7 and day 15. Two groups did not receive any vaccination. All groups except one (unvaccinated, unchallenged) were challenged at day 22 with 250 sporulated E. tenella oocysts. All chickens were weighed and culled five days later. The left caeca were collected immediately and stored in RNAlater ${ }^{\mathrm{TM}}$ (Thermofisher Scientific) at $4{ }^{\circ} \mathrm{C}$ prior to homogenisation.

\section{Studies 2 and 3 (High dose challenge-layer chickens)}

High parasite dose challenge studies were used to assess vaccine efficacy against pathological (e.g. intestinal lesion scoring) and performance (e.g. body weight gain) parameters(21). In study 2, 100 female Hy-Line Brown layer day of hatch chicks were weighed and divided into eight groups of 1213 chicks (Supplementary Table 2). Groups were housed in separate cages, with live oocyst 
vaccinated and unvaccinated/unchallenged groups isolated in separate rooms. In study 3, 210 female Hy-Line Brown layer day of hatch chicks were weighed and divided into six groups of 33-34 chicks, each group was housed in a separate rack of three cages with E. tenella challenged and unchallenged groups isolated in separate rooms (Supplementary Table 2). At day 7 all birds were wing-tagged. Both studies followed the same timetable except that in study 2, one group received a low dose live oocyst "vaccine" of 100 E. tenella oocysts by oral inoculation at day 7 of age.

Both studies received an oral yeast vaccine every 3-4 days from day 7 of age (five doses per chicken in total). In study 2, there were five groups receiving an oral yeast vaccine; empty vector (pYD1 only), pYD1-EtAMA1, pYD1-EtIMP1, pYD1-EtMIC3 or a mixture of pYD1-EtAMA1, pYD1EtIMP1 and pYD1-EtMIC3 (Supplementary Table 2). Two groups did not receive any vaccination. Study 3 included four groups; unvaccinated, unchallenged (-), unvaccinated, challenged (+), empty vector vaccinated (pYD1 only), challenged, and pYD1- All 3 antigens vaccinated, challenged (Supplementary Table 2). All groups except one (negative control group) were challenged at day 22 with 4,000 E. tenella oocysts. The choice of dose level was based upon previous titration in this chicken line (21). All chickens were weighed and culled six days later. The caeca were examined for lesion scores as originally described by (22).

A high parasite dose challenge study was then used to assess vaccine efficacy in broiler lines; as for the work in layers, pathological and performance parameters were evaluated. One hundred and fifty (150) mixed sex Cobb500 broiler day of hatch chicks were initially housed together on fresh litter. At day 7 chickens were weighed, wing-tagged and divided into four groups of 35 chicks, each group was then housed in separate pens with control groups (unvaccinated/unchallenged (-), and unvaccinated/challenged (+)) housed in separate rooms. Two groups received an oral yeast vaccine every 3-4 days from day 7 of age (five doses per chicken in total); the treatments were empty vector (pYD1 only) or a mixture of pYD1-EtAMA1, pYD1-EtIMP1 and pYD1-EtMIC3 (Supplementary Table 2). Due to an outbreak of colibacillosis within all groups, confirmed by bacterial culture of liver samples obtained post-mortem, all chickens were treated with enrofloxacin (Baytril®, Bayer, Leverkusen, Germany) at $10 \mathrm{mg} \mathrm{Kg}^{-1}$ for 3 days from days 16 to 18 . All chickens were weighed at day 21 then all infected groups except one were challenged by oral inoculation with 15, $000 \mathrm{E}$. tenella oocysts. The choice of dose level was based upon previous studies with Cobb500 chickens (23), where a dose higher than that used with the Hy-Line chickens was required to achieve a comparable level of pathology. The unvaccinated/unchallenged negative control group received a mock challenge using PBS. From each group, a randomly selected cohort of 8-10 chickens were weighed and culled six days later to assess the pathological consequences of infection. The caeca from these chickens were examined for lesion scores as originally described by (22). At 10 days post infection the remaining chickens ( $n=19-21$ / group) were weighed and culled. Feeders were emptied at time of challenge and food intake was then measured until chickens were culled to calculate feed conversion ratio (FCR) for each group by dividing total food consumed by total body weight gain (Day 21-31).

\subsection{Isolation of total genomic DNA from caecal tissue for quantification of parasite replication} using the DNeasy Blood and Tissue DNA Kit (QIAGEN) according to manufacturer's instructions. 


\subsection{Quantitative PCR for parasite replication}

294 Quantitative PCR for assessment of E. tenella genome copy number in the caeca was performed as

295

296 previously described to quantify parasite replication (24). Briefly, gDNA purified from caecal tissue was used as template for qPCR targeting E. tenella (RAPD-SCAR marker Tn-E03-116, primers F:TCGTCTTTGGCTGGCTATTC, R: CAGAGAGTCGCCGTCACAGT (25)) and chicken (tatabinding protein (TBP), primers F: TAGCCCGATGATGCCGTAT, R: GTTCCCTGTGTCGCTTGC (26)) genomes. Quantitative PCR was performed in $20 \mu \mathrm{L}$ reactions in triplicate containing $10 \mu \mathrm{L} 2 \times$ SsoFast EvaGreen Supermix (Bio-Rad,), $1 \mu \mathrm{L}$ of primers ( $3 \mu \mathrm{M} F$ and $3 \mu \mathrm{M} \mathrm{R}), 8 \mu \mathrm{L}$ of molecular biological grade water (Invitrogen) and $1 \mu \mathrm{L}$ of gDNA or water as a negative no template control. Hard-shelled 96-well reaction plates (Bio-Rad) were sealed with adhesive film (Bio-Rad) and loaded into a Bio-Rad CFX qPCR cycler. Reactions were heated to $95^{\circ} \mathrm{C}$ for $2 \mathrm{~min}$, prior to 40 cycles consisting of $95^{\circ} \mathrm{C}$ for $15 \mathrm{~s}$ then $60^{\circ} \mathrm{C}$ for $30 \mathrm{~s}$ with a fluorescence reading taken after each cycle. Melting curve analysis was performed consisting of $15 \mathrm{~s}$ at $95^{\circ} \mathrm{C}$, before cooling to $65^{\circ} \mathrm{C}$ for $60 \mathrm{~s}$, then heating to $95^{\circ} \mathrm{C}$ in $0.5^{\circ} \mathrm{C}$ increments for $0.5 \mathrm{~s}$. Absolute quantification was performed against a standard curve generated using serially diluted plasmid DNA containing the amplicon of interest (EtenSCAR or ChickenTBP), to generate a standard curve ranging from $10^{6}$ copies to $10^{1}$ genome copies per $\mathrm{mL}$. Parasite genome copy number was normalised by division with host (chicken) genome copy number.

\subsection{Statistical analysis}

312 Statistical analysis was carried out using GraphPad Prism 8 (Graph Software, LLC). One-way ANOVA was used to compare means of different groups for weight gain and parasite replication, D'Agostino-Pearson normality testing was performed to confirm a Gaussian distribution. The Kruskal-Wallis test was used to compare ranked means of challenge groups for lesion scores. The post-hoc multiple comparison test used for all parameters was Tukeys, and Spearmann rank correlation was used to assess correlations between parameters.

\section{Results}

\subsection{Expression of E. tenella antigens in killed S.cerevisiae}

Confirmation of protein expression by $S$. cerevisiae $24 \mathrm{~h}$ post-induction was provided by antibody staining and flow cytometry, indicating inducible expression of the E. tenella antigens EtAMA1, EtIMP1 or EtMIC3 based upon detection of the 3' V5 epitope tag (examples shown in Supplementary Figure 1). Successful killing of each $S$. cerevisiae vaccine line was confirmed by the absence of growth on (i) minimal dextrose plates supplemented with $1 \%$ leucine and $2 \%$ glucose , and (ii) YNB-CAA medium containing $2 \%$ glucose, after five days incubation. Heat killing at $56{ }^{\circ} \mathrm{C}$ for $1 \mathrm{~h}$ as per study $1 \mathrm{was}$ found to be less consistent in heat inactivating yeast with occasional growth observed compared to the higher temperature of $95{ }^{\circ} \mathrm{C}$ for $2 \mathrm{~min}$, as per study 2 . Thus, the latter treatment was used for subsequent studies. challenge (Study 1)

332 A significant decrease in parasite genome copy number at 5 days post-infection was observed in 333 chickens vaccinated orally with $S$. cerevisiae expressing either EtAMA1Cit or EtIMP1Cit alone, and 
334 for those given an admixture of $S$. cerevisiae expressing both antigens, compared with unvaccinated,

335

336

337

338

339

340

341

342

343

344

345

346

347

348

349

350

challenged chickens $(\mathrm{p}<0.01)$ (Figure 1). The use of a mixture of S.cerevisiae expressing both antigens reduced parasite load significantly compared with S.cerevisiae expressing either EtAMA1Cit ( $<<0.05$ or EtIMP1Cit alone $(p<0.01)$, and compared with live oocyst vaccination $(\mathrm{p}<0.01)$ or vaccination using recombinant EtIMP1 protein $(\mathrm{p}<0.01)$. There was no significant difference in parasite load (number of genomes detected by qPCR) between chickens vaccinated with S.cerevisiae expressing either EtAMA1Cit or EtIMP1Cit alone and those given live oocyst vaccination $(p>0.05)$. Control groups performed as anticipated; there was no significant difference between chickens vaccinated with $S$. cerevisiae containing the empty pYD1 vector $(\mathrm{p}>0.05)$ compared with unvaccinated, challenged chickens. Chickens vaccinated with a live oocyst dose at day 7 or vaccinated with recombinant EtIMP1 protein showed a significant decrease in parasite genome copy number compared with unvaccinated, challenged chickens $(p<0.01)$. Mean percentage reduction in parasite load, compared with unvaccinated, challenged chickens, for chickens vaccinated with $S$. cerevisiae expressing either EtAMA1 or EtIMP1 (64.7\% and $54.7 \%$, respectively) was comparable with vaccination with recombinant EtIMP1 protein given intramuscularly $(59.8 \%)$. Whilst combined vaccination with $S$. cerevisiae expressing EtAMA1 and EtIMP1 reduced mean parasite load further to $86.2 \%$.

\subsection{Caecal lesion scores reduced in a proportion of layer chickens vaccinated with combination of all three antigens expressed in S. cerevisiae in high challenge study (Studies 2 and 3)}

Although there was no statistical difference in mean lesion score between the vaccinated chickens and unvaccinated chickens in Study 2 (Figure 2A), it was apparent that a proportion of chickens that received a mixture of $S$. cerevisiae expressing EtAMA1, EtIMP1 and EtMIC3 had either no visible caecal lesions (5/13) or a lesion score of 1 (3/13). This reduction in lesion score in some chickens was less marked in those which received $S$. cerevisiae expressing only one of the three antigens. In study 3, which studied are larger group of chickens but otherwise followed a comparable study design, a statistically significant reduction in caecal lesion score was observed in groups of chickens vaccinated using a mixture of $S$. cerevisiae expressing EtAMA1, EtIMP1 and EtMIC3 compared with unvaccinated, challenged chickens $(\mathrm{p}<0.01)$ (Figure $2 \mathrm{~B})$. As in study 2, there was marked variability in lesion scores between individual vaccinated chickens (range 0-3).

\subsection{Pre and post E. tenella challenge weight gain in layer chickens was unchanged by E. tenella} infection after low and high dose challenge (Studies 1, 2 and 3)

No significant difference was noted in weight gain between unvaccinated, unchallenged and unvaccinated, challenged Hy-Line layer chickens in studies 1 and 2. Average weight gain in the six days post-challenge was $106.7 \mathrm{~g} \pm 14.02 \mathrm{~g}$ and $85.18 \mathrm{~g} \pm 7.125 \mathrm{~g}$ in the unvaccinated, unchallenged groups, and $97.17 \mathrm{~g} \pm 15.74 \mathrm{~g}$ and $90.67 \mathrm{~g} \pm 11.19 \mathrm{~g}$ in the unvaccinated, challenged groups (studies 1 and 2, respectively). In the absence of a significant difference between positive and negative controls in these studies, weight gain was not assessed as a performance parameter. A statistical difference in weight gain post-challenge was observed in study 3 with the unvaccinated, unchallenged group (78.12 $\mathrm{g} \pm 11.03 \mathrm{~g}$ ) demonstrating higher body weight gain compared with the unvaccinated, challenged group $(65.58 \mathrm{~g} \pm 16.02 \mathrm{~g} ; \mathrm{p}<0.01)$. There was no significant improvement in weight gain post-challenge in $S$. cerevisiae vaccinated chickens compared with unvaccinated, challenged controls in study 3 (65.35 g士 $15.35 \mathrm{~g}$; p>0.05). Chickens used in these studies were commercial layer chickens and there was no anticipated impact on body weight gain in the short time period (5-6 days) studied post-challenge. 
3.5 Caecal lesion scores were reduced in a proportion of broiler chickens vaccinated with a combination of all three S. cerevisiae expressed antigens (Study 4)

As described for the layer chickens, a wider range in lesion score was observed in broiler chickens vaccinated with a mixture of $S$. cerevisiae expressing all three antigens compared to those left unvaccinated (unvaccinated, challenged: average 3.3, range 3-4; test vaccinated, challenged: 2.6, 2-3; Figure 3). However, there was no statistical difference in mean lesion score at day 6 post-challenge (Figure 3).

3.6 Significant increase in body weight gain post E. tenella challenge and improved food conversion ratio following oral yeast vaccination in broiler chickens

A significant increase in body weight gain was observed for Cobb500 chickens vaccinated orally with a mixture of $S$. cerevisiae expressing all three antigens compared with unvaccinated, challenged chickens over the ten days following high level E. tenella challenge ( $<<0.01$; Figure 4$)$. There was also a significant increase in body weight gain in vaccinated chickens compared to mock vaccinated chickens that received the empty pYD1 vector $(\mathrm{p}<0.05)$. There was a significant difference in body weight gain between unvaccinated, unchallenged chickens and unvaccinated, challenged chickens $(\mathrm{p}<0.05)$. When chickens were grouped by sex the significant increase in body weight gain in vaccinated compared with unvaccinated, challenged chickens (study days D21-D31) remained; the increase in body weight was more significant in females $(p<0.001)$ than males $(p<0.05)$. There was no significant difference in body weight gain between groups pre-challenge (D7-21) (p>0.05). There was no significant difference in mean body weight between groups at any of the time points evaluated (D7, D21, D31) ( $\mathrm{p}>0.05)$ (Table 1).

400

Food conversion ratio (FCR) was calculated for each group, together with total body weight gain of chickens culled at six- and ten-days post-challenge (Table 2). FCR was lowest in chickens vaccinated with a mixture of $S$. cerevisiae expressing all three antigens at 1.52, comparable to unvaccinated, unchallenged chickens with an FCR of 1.56. Chickens vaccinated with $S$. cerevisiae with empty pYD1-vector had a higher FCR of 1.65 comparable to unvaccinated, challenged chickens with a FCR of 1.67. Statistical comparison of the differences in FCR between groups was not possible because values were calculated per treatment group, rather than for individual chickens.

\section{Discussion}

Development of novel recombinant or subunit vaccines against Eimeria species in chickens has been limited thus far. Despite many promising pilot studies with various antigens, none have progressed to commercial products (27). The absence of an efficacious, cost-effective and scalable system for routine vaccination of broilers remains a persistent challenge for Eimeria, as well as other pathogens. In this study we sought to address some of these barriers to commercialisation for Eimeria vaccines by developing an oral inactivated whole yeast-based vaccine which could be produced easily and cheaply, and potentially be administered in-feed. Taking a panel of candidate immunoprotective antigens validated previously using recombinant protein and/or DNA vaccination screens (4-6), we have demonstrated here that $S$. cerevisiae yeast expressing E. tenella antigens could be produced and delivered as a whole inactivated yeast vaccine safely to layer and broiler chickens. Further, such vaccines can be effective in reducing $E$. tenella replication in the caeca, reduce intestinal lesion score, and improve body weight gain and food conversion post-challenge. Saccharomyces cerevisiae expressing E. tenella antigens were heat killed and freeze-dried before use as a whole yeast vaccine, 
thus they were no longer classified as genetically modified organisms (GMO) which simplifies future licensing. Heat-killing of $S$. cerevisiae has been described elsewhere $(15,28)$, although there is little published data on validating methods of heat-killing for yeast and specification by national/international regulators will likely be needed. From this study, it appeared that heat killing at high temperature $\left(95^{\circ} \mathrm{C}\right)$ for two minutes was more reliable than a longer incubation at lower temperature, but this may vary depending on the concentration of yeast particles incubated and method of heating. Previous studies have demonstrated that protein antigen stability in yeast can be maintained for up to a year even at room temperature, making this system ideally suited for use in developing countries where cold-chain access may be limited (29). Based on previous studies heatkilling does not appear to impact immunogenicity (reviewed in (30)), although this was not within the scope of our study.

Vaccination with $S$. cerevisiae expressing EtAMA1 and EtIMP1, alone or together, was successful in reducing parasite replication following low level E. tenella challenge. Parasite replication was assessed in the context of a low level parasite challenge since the Eimeria crowding effect(19) can be expected to obscure partial protective responses at higher levels of challenge, as illustrated in a recent dose titration study using layer-breed chickens(21). Yeast vaccination with a single antigen was comparable to vaccination by injection with the equivalent recombinant EtIMP1 protein in the low dose challenge. Previous studies have demonstrated the efficacy of these antigens when delivered subcutaneously or intramuscularly as protein or DNA vaccines (4-6). More recent studies have delivered these antigens orally $(31,32)$. EtAMA1 and EtIMP1 expressed and delivered in a live recombinant bacterial Lactococcus lactis vaccine resulted in reductions in oocyst output and lesion score $(31,32)$. Similarly, EtAMA1 and EtMIC2 co-expressed in Lactobacillus plantarum and delivered as an oral vaccine reduced oocyst output and lesion score following challenge (33). As noted for most recombinant antigen-based vaccines for Eimeria (reviewed in (27)), our yeast-based vaccine reduced parasite replication but did not completely prevent parasite development and shedding. Modern ionophore anticoccidial formulations reduce replication of drug susceptible Eimeria populations by $82-97 \%$ (34) and it is likely that a successful recombinant vaccine will need to achieve a comparable reduction. It is noteworthy that vaccination using a mixture of yeast expressing EtAMA1 with EtIMP1 achieved $86.2 \%$ reduction in parasite replication, which is in the range of commercially viable levels of efficacy. Moreover, in a field situation, reduction in parasite replication and oocyst output is likely to be boosted by low levels of parasite escape and recycling as seen with current live and live-attenuated vaccines, as well as with the use of current anticoccidial drugs (3). Furthermore, it will likely be possible to improve vaccine efficacy by altering doses or treatment schedules, which will be examined in future studies.

Assessment of the effects of Eimeria infection on enteric pathology and production parameters require high levels of parasite challenge, carefully titrated to achieve a measurable phenotype without mortality (21). Performance parameters such as body weight gain and FCR can most usefully be assessed in broiler-type chickens that have rapid and efficient growth (35). Slower growing layertype chickens are less likely to provide discriminatory phenotypes in a short study, as seen here using Hy-Line Brown layer chickens. The results of our high challenge broiler study indicate significant improvement in key production traits of body weight gain and food conversion rates in chickens vaccinated with $S$. cerevisiae expressing E. tenella antigens, suggesting that disease was sufficiently reduced to allow for sustained body weight gain required by the broiler industry. Indeed, average body weight gain post-challenge in S. cerevisiae- E. tenella vaccinated broilers was higher than in unchallenged chickens, better even than the $90 \%$ body weight maintenance observed with monensin treated chickens reported previously (34). This difference might reflect compensatory growth, or a positive additive effect of dietary supplementation using $S$. cerevisiae $(10,11)$. Reducing the 
468

469

470

471

472

473

474

475

476

477

478

479

480

481

482

483

484

485

486

487

488

489

490

491

492

493

494

495

496

497

498

499

500

501

502

503

504

505

506

507

508

509

510

511

512

513

consequences of Eimeria infection can also be expected to improve chicken welfare. Further field studies are required to evaluate the use of this vaccine under commercial broiler conditions to confirm its applicability to the market.

While yeast-vectored anticoccidial vaccination improved measures of parasite replication and broiler performance, the impact of vaccination on lesion score was more nuanced. Considerable inter-animal variation was observed in lesion scores in vaccinated chickens, with some reductions statistically significant while others were not. Lesion scores were also subjectively higher within the broiler study compared to the high challenge layer studies, reflecting differences in genetic resistance/susceptibility between these chicken types. Development of lesions following Eimeria infection is complex and is likely a combination of host genetics, level of parasite damage to epithelial cells and host inflammatory response (36). Genome-wide association studies (GWAS) have suggested a significant host genetic influence on the outcome of secondary Eimeria infection (37), a feature that might also apply to the success of vaccination. It has been suggested that lesions deriving from primary infection might suggest severe disease, whilst lesions in chickens vaccinated with live or live attenuated vaccines arising post vaccination or following subsequent challenge are not necessarily indicative of a lack of protection from disease $(35,38)$. Previous studies have demonstrated the presence of lesions in chickens vaccinated with live attenuated vaccines, however lesions were less severe in vaccinated chickens and not always associated with presence of endogenous parasites compared to unvaccinated chickens where parasite numbers were high (39). Although our study did not microscopically examine caecal lesions, evidence of reduced parasite replication in the caeca as demonstrated by the qPCR data could support a similar phenomenon following vaccination with our yeast vaccines.

In addition to antigen expression and delivery, a killed yeast vaccine can also provide an immunostimulatory adjuvating effect. It is well established that $S$. cerevisiae yeast are immunogenic and can be taken up and activate macrophages and dendritic cells (40) through receptors such as the mannose receptor and Dectin-1, which also have been shown to be expressed on mammalian M-cells (41). Whilst this mechanism makes them ideal for generating an antigen-specific adaptive immune response following antigen presentation through $\mathrm{MHC}$, they also stimulate an innate immune response. Indeed, studies feeding yeast cell-wall components to chickens infected with Eimeria have demonstrated reduction in parasite replication and improvement in production traits (9-11). In the broiler study (Study 4), the improvement in body weight gain observed post-challenge appeared to be independent of the "yeast effect" with a significant increase in chickens vaccinated with S. cerevisiae expressing E. tenella antigens compared with those given $S$. cerevisiae empty vector control. Nonetheless, the beneficial effects of dietary yeast supplementation can add value to a vectored anticoccidial vaccine; dose optimisation will likely be required.

Any novel Eimeria vaccine will need to incorporate antigens that stimulate immune responses protective against more than one Eimeria species for it be a viable alternative to anticoccidial drugs or existing live and live-attenuated vaccines. It is well established that there is little to no cross immune protection against heterologous challenge between Eimeria species, indicating a requirement for additional antigens(42). Eimeria tenella was selected for initial proof of concept being both well described with established infection models and also an important species in terms of prevalence and pathogenicity $(17,43)$. Eimeria tenella is also recognised as one of the least immunogenic of the Eimeria that infect chickens (18), which suggests that protection achieved here could be improved when using equivalent antigens derived from other, more immunogenic, species. All current live anticoccidial vaccines target Eimeria acervulina and E. maxima, in addition to E. tenella, as a core unit (44). Some vaccines formulated for broiler chicken markets have established a more specific 
514 identity by inclusion of other species such as E. mitis (e.g. Paracox-5 or HuveGuard MMAT) as well

515 as E. praecox (e.g. Evant). Species such as E. brunetti and E. necatrix are usually only required in

516 vaccines for longer lived layer or breeder chickens (44). Future studies should focus on the addition

517 of antigens from Eimeria acervulina and E. maxima, especially important in North America (45),

518 which in combination with E. tenella would represent the three species most costly to global chicken

519 production.

520 In conclusion we have demonstrated that a heat killed oral $S$. cerevisiae vaccine expressing E. tenella antigens is safe and effective in reducing parasite replication following challenge with E. tenella. Future work should extend examination of the impact of vaccination on production traits such as body weight gain and food conversion ratio in broiler chickens during challenge by E. tenella as well as other key Eimeria species to ensure this approach is a viable alternative to anticoccidial drugs.

525

\section{Conflict of Interest}

527

528

529

530

The authors declare no conflict of interest. The funders had no role in the design of the study; in the collection, analyses, or interpretation of data; in the writing of the manuscript, or in the decision to publish the results.

\section{Author Contributions}

Conceptualisation, D.B., D.W. and F.T.; methodology, F.S., D. B., M.N. and T.K..; validation, F.S. and D.B. formal analysis, F.S.; investigation, F.S., M.N. and T.K.; writing-original draft preparation, F.S.; writing-review and editing, D.B., D.W. and F.T.; visualisation, F.S.; supervision, D.B., D.W. and F.T.; project administration, F.S., E.A., V.M-H. and S.K ; funding acquisition, D.B., D.W. and F.T. All authors have read and agreed to the published version of the manuscript.

\section{Funding}

This study was funded by the Biotechnology and Biological Sciences Research Council (BBSRC) through grant BB/P003931/1 and by The Bloomsbury SET through grant BSA36.

\section{Acknowledgments}

The authors would like to thank Iván Pastor-Fernández, Kelisandia Aguiar-Martins, Gonzalo Sanchez Arsuaga, Eleanor Karp-Tatham and Michelle Jones for assistance with animal studies.

The data presented in this study are available on request from the corresponding author. 
551 1. Blake DP, Knox J, Dehaeck B, Huntington B, Rathinam T, Ravipati V, et al. Re-calculating

552 the cost of coccidiosis in chickens. Vet Res. 2020;51(1):115.

5532 2. Williams RB, Carlyle WW, Bond DR, Brown IA. The efficacy and economic benefits of

554 Paracox, a live attenuated anticoccidial vaccine, in commercial trials with standard broiler chickens

555 in the United Kingdom. Int J Parasitol. 1999;29(2):341-55.

556 3. Williams RB, Gobbi L. Comparison of an attenuated anticoccidial vaccine and an

557 anticoccidial drug programme in commercial broiler chickens in Italy. Avian Pathol. 2002;31(3):253-

55865.

559 4. Lai L, Bumstead J, Liu Y, Garnett J, Campanero-Rhodes MA, Blake DP, et al. The Role of

560 Sialyl Glycan Recognition in Host Tissue Tropism of the Avian Parasite Eimeria tenella. Plos

561 Pathogens. 2011;7(10).

562 5. Pastor-Fernández I, Kim S, Billington K, Bumstead J, Marugan-Hernandez V, Kuster T, et al.

563 Development of cross-protective Eimeria-vectored vaccines based on apical membrane antigens. Int

564 J Parasitol. 2018;48(7):505-18.

565 6. Blake DP, Billington KJ, Copestake SL, Oakes RD, Quail MA, Wan KL, et al. Genetic

566 mapping identifies novel highly protective antigens for an apicomplexan parasite. PLoS Pathog.

$567 \quad 2011 ; 7(2): \mathrm{e} 1001279$.

568 7. Gellissen G, Melber K, Janowicz ZA, Dahlems UM, Weydemann U, Piontek M, et al.

569 Heterologous protein production in yeast. Antonie Van Leeuwenhoek. 1992;62(1-2):79-93.

$570 \quad$ 8. Alizadeh M, Rodriguez-Lecompte JC, Rogiewicz A, Patterson R, Slominski BA. Effect of 571 yeast-derived products and distillers dried grains with solubles (DDGS) on growth performance, gut 572 morphology, and gene expression of pattern recognition receptors and cytokines in broiler chickens. 573 Poult Sci. 2016;95(3):507-17.

574 9. Awais MM, Jamal MA, Akhtar M, Hameed MR, Anwar MI, Ullah MI. Immunomodulatory 575 and ameliorative effects of Lactobacillus and Saccharomyces based probiotics on pathological effects 576 of eimeriasis in broilers. Microb Pathog. 2019;126:101-8.

577 10. Gao J, Zhang HJ, Wu SG, Yu SH, Yoon I, Moore D, et al. Effect of Saccharomyces

578 cerevisiae fermentation product on immune functions of broilers challenged with Eimeria tenella.

579 Poult Sci. 2009;88(10):2141-51.

580 11. Lensing M, van der Klis JD, Yoon I, Moore DT. Efficacy of Saccharomyces cerevisiae 581 fermentation product on intestinal health and productivity of coccidian-challenged laying hens. Poult 582 Sci. 2012;91(7):1590-7.

583 12. Chen P, Lv J, Zhang J, Sun H, Chen Z, Li H, et al. Evaluation of immune protective efficacies 584 of Eimeria tenella EtMic1 polypeptides with different domain recombination displayed on yeast 585 surface. Exp Parasitol. 2015;155:1-7.

586 13. Sun H, Wang L, Wang T, Zhang J, Liu Q, Chen P, et al. Display of Eimeria tenella EtMic2

587 protein on the surface of Saccharomyces cerevisiae as a potential oral vaccine against chicken

588 coccidiosis. Vaccine. 2014;32(16):1869-76. 
589

590

591

592

593

594

595

596

597

598

599

600

601

602

603

604

605

606

607

608

609

610

611

612

613

614

615

616

617

618

619

620

621

622

623

624

625

626

627

628

629

14. Roohvand F, Shokri M, Abdollahpour-Alitappeh M, Ehsani P. Biomedical applications of yeast- a patent view, part one: yeasts as workhorses for the production of therapeutics and vaccines. Expert Opin Ther Pat. 2017;27(8):929-51.

15. Bazan SB, Breinig T, Schmitt MJ, Breinig F. Heat treatment improves antigen-specific T cell activation after protein delivery by several but not all yeast genera. Vaccine. 2014;32(22):2591-8.

16. Bumstead JM. Eimeria tenella microneme protein 3: Localisation and function during invasion. University Of Keele; 2008.

17. Reid AJ, Blake DP, Ansari HR, Billington K, Browne HP, Bryant J, et al. Genomic analysis of the causative agents of coccidiosis in domestic chickens. Genome Res. 2014;24(10):1676-85.

18. Long PL, Millard BJ, Joyner LP, Norton CC. A guide to laboratory techniques used in the study and diagnosis of avian coccidiosis. Folia Vet Lat. 1976;6(3):201-17.

19. Williams RB. Quantification of the crowding effect during infections with the seven Eimeria species of the domesticated fowl: its importance for experimental designs and the production of oocyst stocks. International Journal for Parasitology. 2001;31(10):1056-69.

20. Bumstead JM, Bumstead N, Rothwell L, Tomley FM. Comparison of immune responses in inbred lines of chickens to Eimeria maxima and Eimeria tenella. Parasitology. 1995;111 ( Pt 2):14351.

21. Soutter F, Werling D, Kim S, Pastor-Fernández I, Marugán-Hernández V, Tomley FM, et al. Impact of Eimeria tenella Oocyst Dose on Parasite Replication, Lesion Score and Cytokine Transcription in the Caeca in Three Breeds of Commercial Layer Chicken. Front Vet Sci. 2021;8:640041.

22. Johnson J, Reid WM. Anticoccidial drugs: lesion scoring techniques in battery and floor-pen experiments with chickens. Exp Parasitol. 1970;28(1):30-6.

23. Boulton K, Nolan MJ, Wu Z, Psifidi A, Riggio V, Harman K, et al. Phenotypic and genetic variation in the response of chickens to Eimeria tenella induced coccidiosis. Genet Sel Evol. 2018;50(1):63.

24. Nolan MJ, Tomley FM, Kaiser P, Blake DP. Quantitative real-time PCR (qPCR) for Eimeria tenella replication--Implications for experimental refinement and animal welfare. Parasitol Int. 2015;64(5):464-70.

25. Blake DP, Qin Z, Cai J, Smith AL. Development and validation of real-time polymerase chain reaction assays specific to four species of Eimeria. Avian Pathol. 2008;37(1):89-94.

26. Li YP, Bang DD, Handberg KJ, Jorgensen PH, Zhang MF. Evaluation of the suitability of six host genes as internal control in real-time RT-PCR assays in chicken embryo cell cultures infected with infectious bursal disease virus. Vet Microbiol. 2005;110(3-4):155-65.

27. Blake DP, Pastor-Fernandez I, Nolan MJ, Tomley FM. Recombinant anticoccidial vaccines a cup half full? Infect Genet Evol. 2017;55:358-65.

28. Liu M, Clemons KV, Bigos M, Medovarska I, Brummer E, Stevens DA. Immune responses induced by heat killed Saccharomyces cerevisiae: a vaccine against fungal infection. Vaccine. 2011;29(9):1745-53.

29. Kumar R. Investigating the long-term stability of protein immunogen(s) for whole recombinant yeast-based vaccines. FEMS Yeast Res. 2018;18(7). 
30. Franzusoff A, Duke RC, King TH, Lu Y, Rodell TC. Yeasts encoding tumour antigens in cancer immunotherapy. Expert Opin Biol Ther. 2005;5(4):565-75.

632 31. Li J, Wang F, Ma C, Huang Y, Wang D, Ma D. Recombinant lactococcus lactis expressing

633 Eimeria tenella AMA1 protein and its immunological effects against homologous challenge. Exp

634 Parasitol. 2018;191:1-8.

635 32. Ma C, Li G, Chen W, Jia Z, Yang X, Pan X, et al. Eimeria tenella: IMP1 protein delivered by

636 Lactococcus lactis induces immune responses against homologous challenge in chickens. Vet

637 Parasitol. 2021;289:109320.

638 33. Liu Q, Jiang Y, Yang W, Liu Y, Shi C, Liu J, et al. Protective effects of a food-grade

639 recombinant Lactobacillus plantarum with surface displayed AMA1 and EtMIC2 proteins of Eimeria 640 tenella in broiler chickens. Microb Cell Fact. 2020;19(1):28.

641 34. Chapman HD. Eimeria tenella in chickens: Studies on resistance to the anticoccidial drugs 642 monensin and lasalocid. Veterinary Parasitology. 1976;2(2):187-96.

643 35. Williams RB, Catchpole J. A new protocol for a challenge test to assess the efficacy of live 644 anticoccidial vaccines for chickens. Vaccine. 2000;18(13):1178-85.

645 36. Long PL, Johnson J, Wyatt RD. Eimeria-Tenella - Clinical Effects in Partially Immune and 646 Susceptible Chickens. Poultry Sci. 1980;59(10):2221-4.

647 37. Boulton K, Nolan MJ, Wu Z, Riggio V, Matika O, Harman K, et al. Dissecting the Genomic 648 Architecture of Resistance to Eimeria maxima Parasitism in the Chicken. Front Genet. 2018;9:528.

649 38. Williams RB, Andrews SJ. The origins and biological significance of the coccidial lesions 650 that occur in chickens vaccinated with a live attenuated anticoccidial vaccine. Avian Pathology. $6512001 ; 30(3): 215-20$.

652 39. Williams RB. Anticoccidial vaccination: the absence or reduction of numbers of endogenous 653 parasites from gross lesions in immune chickens after virulent coccidial challenge. Avian Pathol. $654 \quad 2003 ; 32(5): 535-43$.

655 40. Stubbs AC, Martin KS, Coeshott C, Skaates SV, Kuritzkes DR, Bellgrau D, et al. Whole recombinant yeast vaccine activates dendritic cells and elicits protective cell-mediated immunity. Nat Med. 2001;7(5):625-9.

658 41. Rochereau N, Drocourt D, Perouzel E, Pavot V, Redelinghuys P, Brown GD, et al. Dectin-1 659 is essential for reverse transcytosis of glycosylated SIgA-antigen complexes by intestinal M cells. $660 \quad$ PLoS Biol. 2013;11(9):e1001658.

661 42. Shirley MW, Smith AL, Tomley FM. The biology of avian Eimeria with an emphasis on their 662 control by vaccination. Adv Parasitol. 2005;60:285-330.

663 43. Chapman HD, Shirley MW. The Houghton strain of Eimeria tenella: a review of the type 664 strain selected for genome sequencing. Avian Pathol. 2003;32(2):115-27.

665 44. Chapman HD. Practical use of vaccines for the control of coccidiosis in the chicken. World's 666 Poultry Science Journal. 2000;56(1):7-20.

667 45. Gilbert W, Bellet C, Blake DP, Tomley FM, Rushton J. Revisiting the economic impacts of 668 Eimeria and its control in European intensive broiler systems with a recursive modelling approach. $6692020 ; 7(558182)$. 


\section{11. Figure Captions}

672 Figure 1. Eimeria tenella replication assessed by qPCR of genomic DNA extracted from caeca

673 following low dose challenge in Hy-line brown layer chickens (Study 1). Treatment groups are

674 shown on the x-axis and E. tenella genome copy number corrected using chicken TBP copy number

675 is on the y-axis. Each marker represents one chicken ( $n=6-8$ per group). Mean and standard

676 deviation for each group is shown. Groups with significantly different mean corrected parasite copy

677 number compared with unvaccinated, challenged chicken group are shown with letter a. The group

678 with significantly mean corrected parasite copy number compared with all other groups is shown

679 with letters ab. Percentage reduction in mean corrected parasite copy number compared with

680 unvaccinated, challenged chicken group are shown above graph.

681 Figure 2. Caecal lesion scores six days post high dose $E$. tenella infection in Hy-line brown

682 layer chickens (Studies 2 and 3) Treatment groups are shown on the x-axis and lesion scores 683 are shown on the y-axis. Each marker represents one chicken (Study 2: $n=10-13$, Study 3: $n=33$ -

684 34). A. Caecal lesion scores for study 2. B. Caecal lesion scores for study 3. Groups with 685 significantly different mean lesion score compared with the equivalent unvaccinated, challenged 686 chicken group are shown with an asterisk $(*)$.

687 Figure 3 Caecal lesion scores six days post high dose $E$. tenella challenge in Cobb500 688 broiler chickens (Study 4). Treatment groups are shown on the $\mathrm{x}$-axis and lesion scores are 689 shown on the $y$-axis. Each marker represents one chicken $(n=8-10)$.

690 Figure 4 Body weight gain post high dose $E$. tenella challenge in Cobb500 broiler chickens 691 (Study 4). Treatment groups are shown on the x-axis and body weight gain in grams from Day 692 21-31 is shown on the y-axis. Each marker represents one chicken ( $n=19-21$ per group). Groups 693 with significantly different body weight gain compared with the unvaccinated, challenged 694 chicken group are denoted by the letter a and those significantly different from empty vector 695 (pYD1 only) vaccinated challenge group denoted by the letter b. One asterisk $(*)$ denotes 696 significance level $\mathrm{p}<0.05$, two asterisk (**) denotes significance level $\mathrm{p}<0.01$ (One way 697 ANOVA, Tukey multiple comparison correction). 
12. Tables

\section{Table 1. Summary of Cobb500 broiler chicken body weight in the high dose E.tenella challenge study}

701 (Study 4). Chickens were weighed at day 7 (pre-vaccination), day 21 (day of challenge) and day 31 (10 days

702 post-challenge).

\begin{tabular}{|c|c|c|c|c|c|}
\hline \multirow[b]{2}{*}{ Group } & \multicolumn{3}{|c|}{ Mean body weight(g), (SD) } & \multicolumn{2}{|c|}{ Mean body weight gain $(\mathrm{g})$} \\
\hline & Day 7 & Day 21 & Day 31 & $\begin{array}{l}\text { Pre- } \\
\text { challenge(D7-21) }\end{array}$ & $\begin{array}{l}\text { Post- } \\
\text { challenge(D21-31) }\end{array}$ \\
\hline Unvaccinated, challenged $(+)$ & $\begin{array}{l}212 \\
(13.58)\end{array}$ & $\begin{array}{l}1139(112 . \\
2)\end{array}$ & $\begin{array}{l}2036 \\
(191.3)\end{array}$ & $926.9(103.9)$ & $916.3(123.5)$ \\
\hline Unvaccinated, unchallenged (-) & $\begin{array}{l}216.1 \\
(15.23)\end{array}$ & $\begin{array}{l}1149 \\
(124.2)\end{array}$ & $\begin{array}{l}2172 \\
(263.3)\end{array}$ & $933(114.7)$ & $1058(150.6)^{a}$ \\
\hline $\begin{array}{l}\text { Empty vector vaccinated(pYD1 only), } \\
\text { challenged }\end{array}$ & $\begin{array}{l}208.3 \\
(18.38)\end{array}$ & $\begin{array}{l}1112(107 . \\
2)\end{array}$ & $\begin{array}{l}2094 \\
(300)\end{array}$ & $903.8(100.9)$ & $977.1(193.4)$ \\
\hline pYD1-All 3 antigens, challenged & $\begin{array}{l}211.6 \\
(15.05)\end{array}$ & $\begin{array}{l}1108 \\
(116.1)\end{array}$ & $\begin{array}{l}2223 \\
(216.2)\end{array}$ & $896.8(108.4)$ & $1105(124.1)^{\mathrm{ab}}$ \\
\hline Number chickens per group & $28-32$ & $28-32$ & $19-21$ & $28-32$ & 19-21 \\
\hline F statistic, $p$ value (ANOVA) & $1.261,0.29$ & $\begin{array}{l}0.8866, \\
0.45\end{array}$ & $\begin{array}{l}2.319, \\
0.08\end{array}$ & $0.7825,0.51$ & $6.317,0.0007$ \\
\hline
\end{tabular}

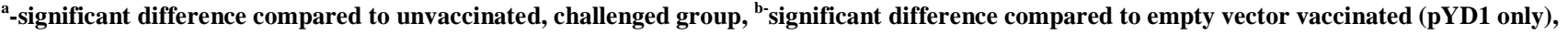
challenged group 
706 Table 2. Summary of food conversion ratio (FCR) in Cobb500 broiler chickens in the high dose $E$.

707 tenella challenge study (Study 4). FCR was calculated together with total body weight gain of

708 chickens culled at six and ten days post-challenge.

\begin{tabular}{|c|c|c|c|}
\hline & $\begin{array}{c}\text { Total } \\
\text { weight gain } \\
\text { post- } \\
\text { challenge } \\
\text { (kg) D21- } \\
\text { D31 }\end{array}$ & $\begin{array}{c}\text { Total food } \\
\text { consumed(kg)D21- } \\
\text { D31 }\end{array}$ & $\begin{array}{c}\text { Food } \\
\text { conversion } \\
\text { ratio }\end{array}$ \\
\hline Unvaccinated, challenged(+) & 25.171 & 42.14 & 1.67414882 \\
\hline Unvaccinated, unchallenge(-) & 25.174 & 39.23 & 1.55835386 \\
\hline $\begin{array}{c}\text { Empty vector vaccinated (pYD1 only), } \\
\text { challenged }\end{array}$ & 26.653 & 44.00 & 1.65084606 \\
\hline pYD1-All 3 antigens, challenged & 26.177 & 39.78 & 1.51965466 \\
\hline
\end{tabular}




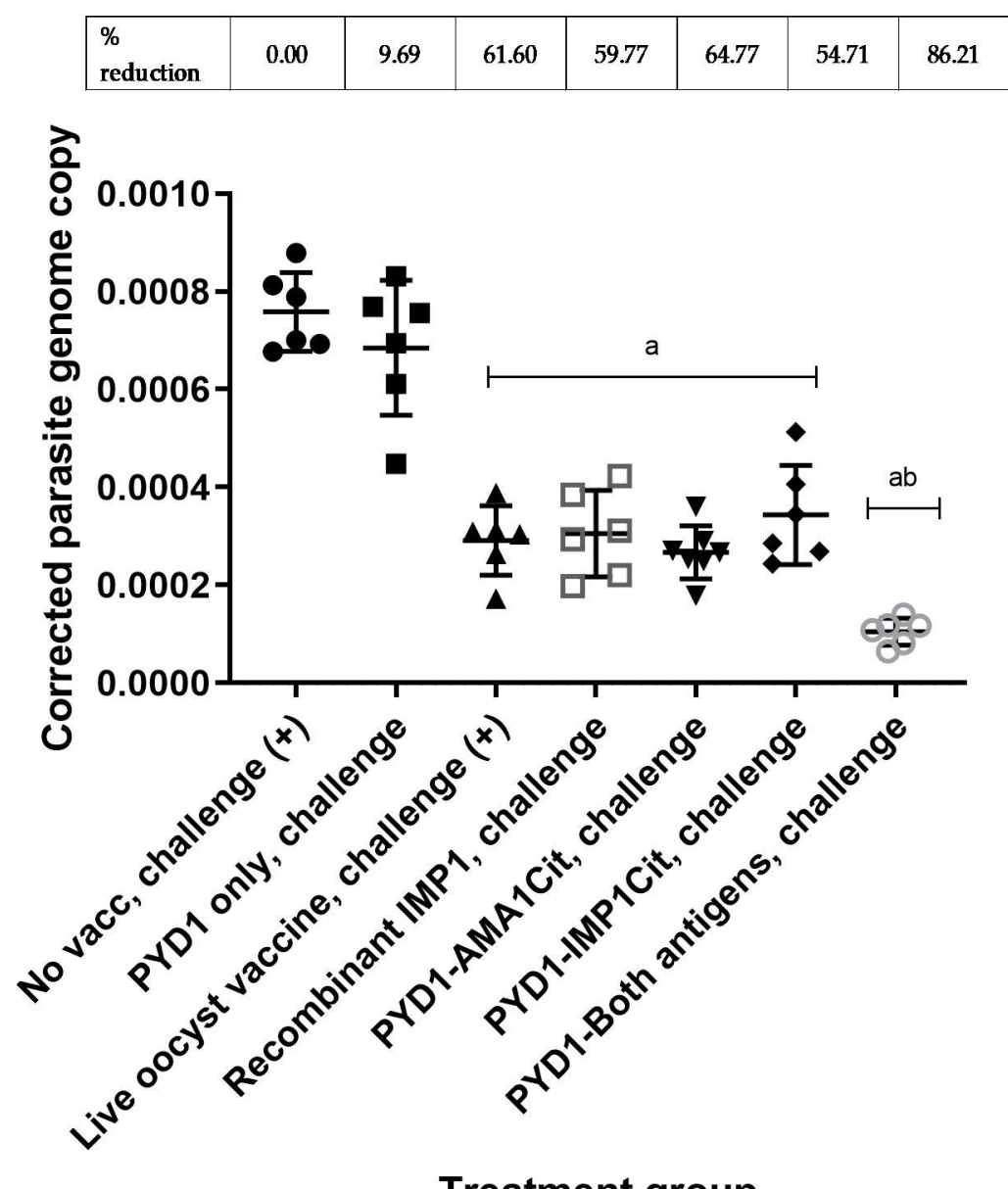

Treatment group 
A
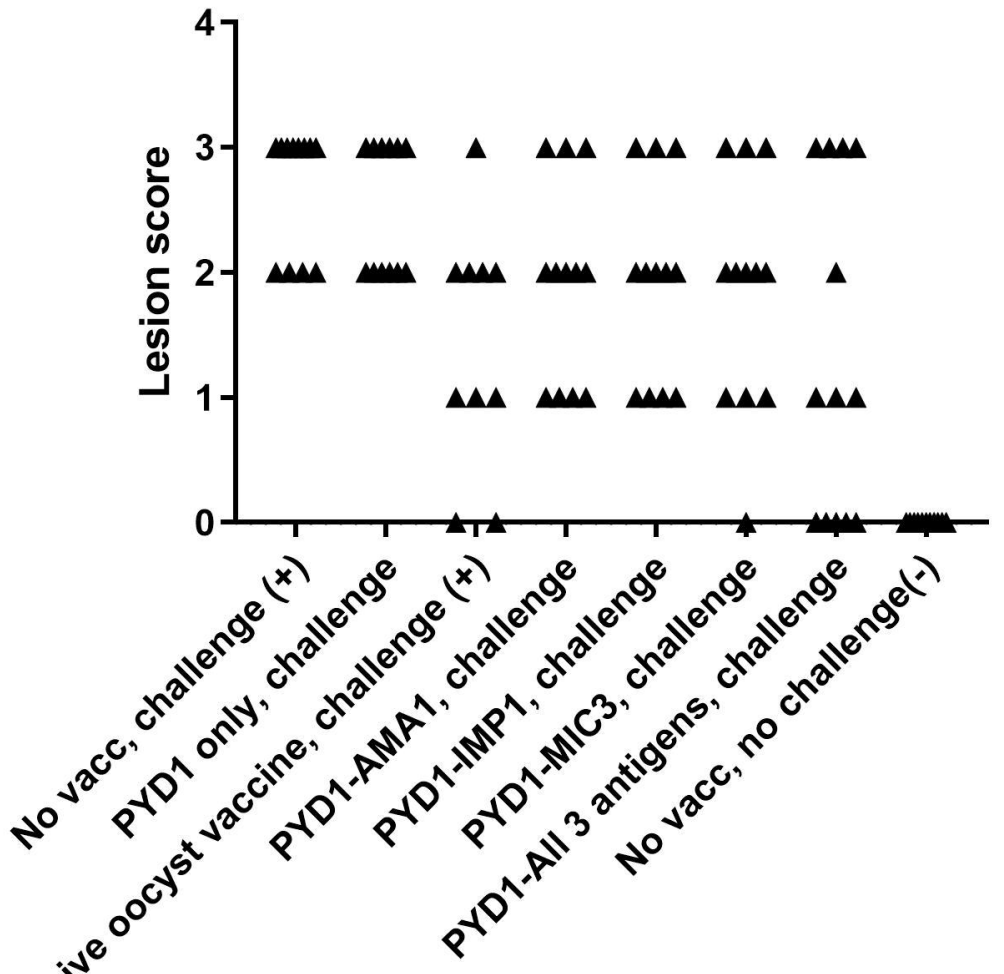

Treatment Group 
B

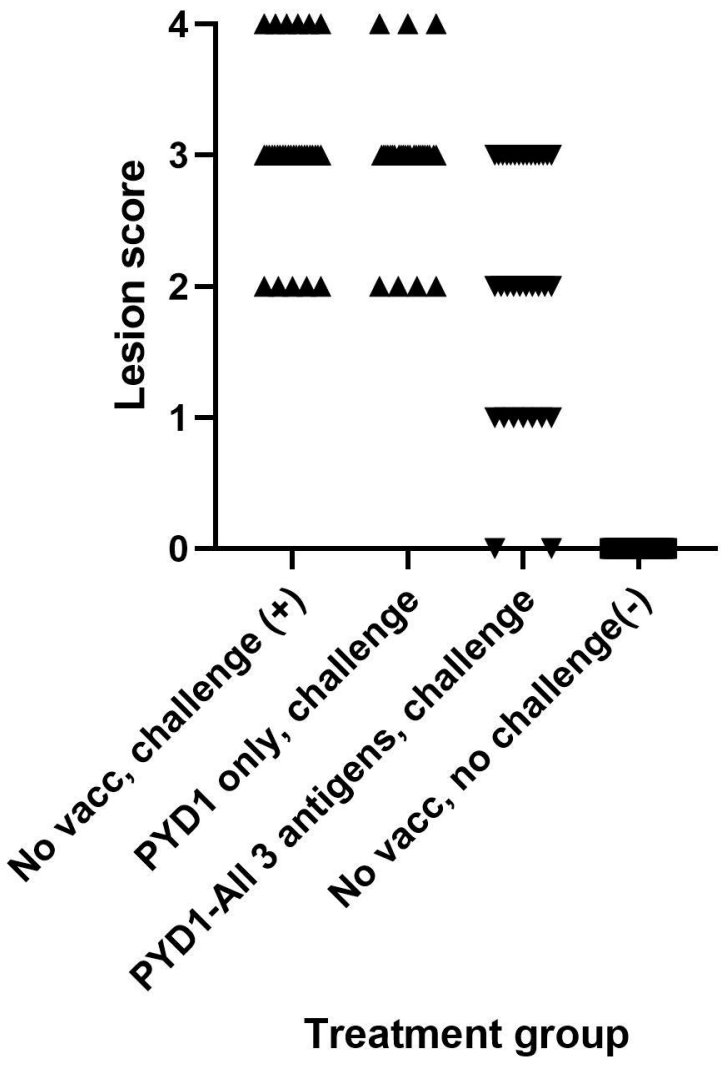




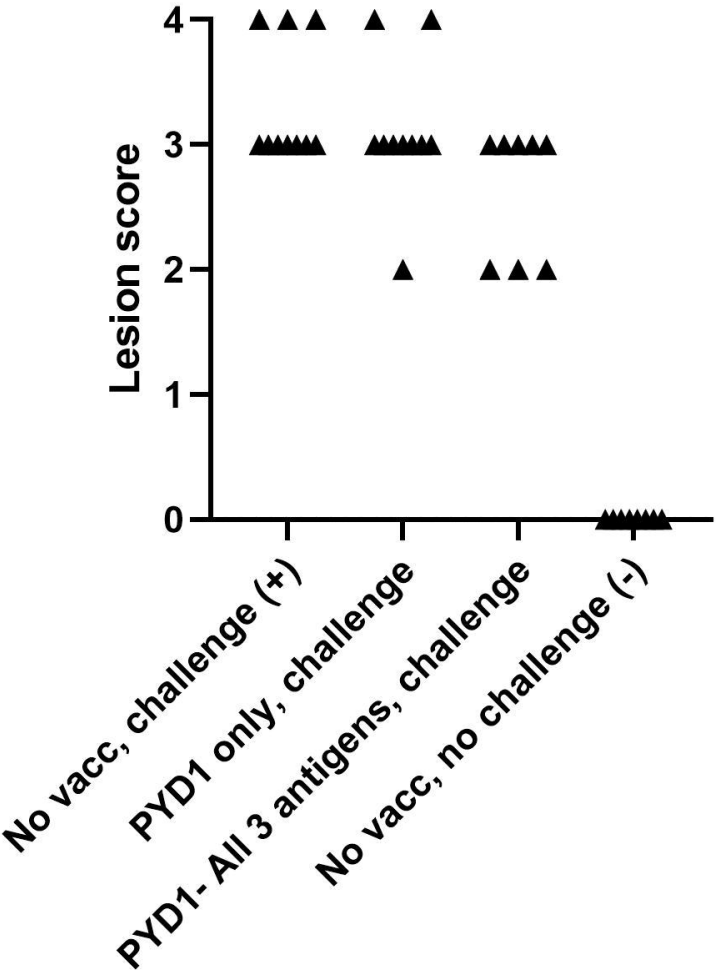




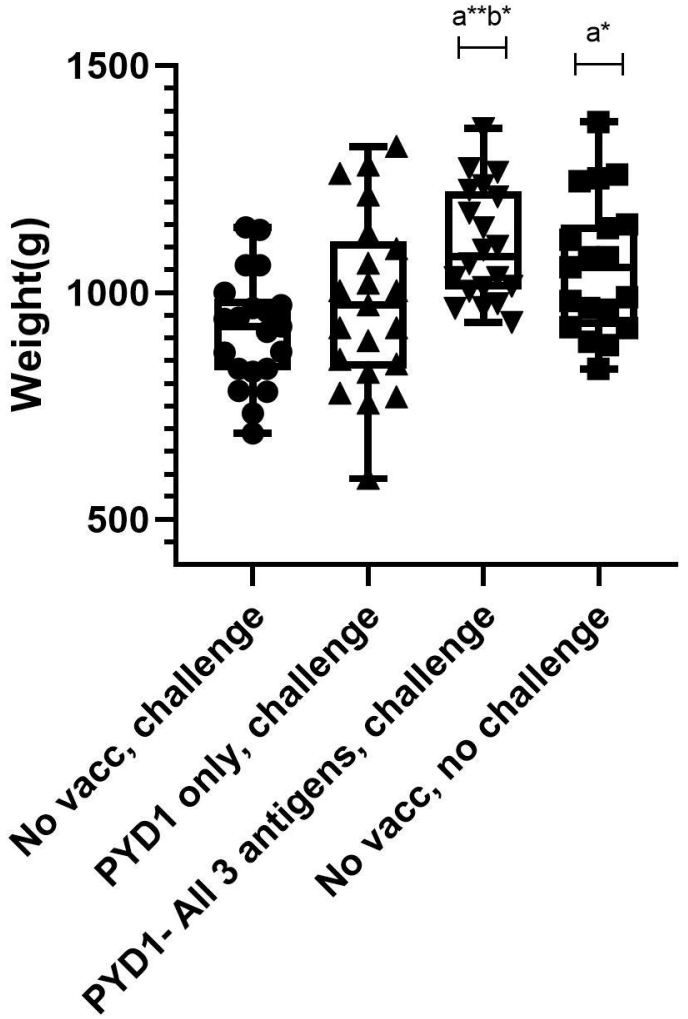

